Se terminó de imprimir este libro el día 22 de enero de 1975 en los talleres de la Editorial Libros de México, S. A., Av. Coyoacán 1035, México 12. D. F. Su tiro consta de 1600 ejemplares.

No 0871 



\section{MESTER}

Revista de los estudiantes graduados del Departamento de Español

y Portugués de la Universidad de California en Los Angeles.

Se publica dos veces por año, en primavera y en otoño. Su enfoque es la creación literaria y el estudio de escritores iberoamericanos, españoles y portugueses.

Se les da preferencia a los escritores de nuestra época.

\section{Suscripciones y ventas:}

Número suelto .......... 3.00 dólares

Suscripción anual ......... 6.00 dólares

Suscripción de estudiante .... 4.00 dólares

Toda correspondencia debe dirigirse a :

\section{Roberto Cantú}

Mester

Department of Spanish and Portuguese

University of California

Los Angeles, Calif. 90024

USA

FOREIGN BOOKS AND PERIODICALS
CURRENT OR OUT-OF-PRINT
Specialties:
Search Service
Irregular Serials
International Congresses
Building Special Collections
ALBERT J. PHIEBIG, INC.
Box 352, White Plains, N. Y. 10602




\section{TERZOMO N D O}

Rivista trimestral di studi, ricerche e documentazione

sui paesi afro-asiatici e latino-americani

Diretta da Umberto Melotti

Anno VI No. $19-20$

Marzo-Giugno 1973

SOMMARIO: Commenti, Giovanni Bianchi, Cristiani e internazionalismo operaio - Saggi, Samir Amin, Per una strategia alternativa di sviluppo autocentrato - Note Mario Aglieri Rinella, La cooperazione cinese con i paesi in via di sviluppo - Rassegne, Marco Ingrosso, Modelli socioeconomici di interpretazione della realtà latino-americana: da Mariátegui ad André Gunder Frank - I nostri temi, Luciano Pellicani, Le rivoluzioni del Terzo Mondo.

TERZO MONDO: Direzione, Redazione, Amministrazione: Via G.

B. Morgagni 39 - 20129 Milano - Tel. 209-041. ABBONAMENTI 1973 ordinario L. 4000 , d'amicizia L. 5000 , sostenitore L. 10000 Versamenti sul conto conente postale n $3 / 56111$ o con assegno. SUBSGRIPTION/ABONNEMENT/SUSGRIPGION US $\$ 7$ - By bank or money-orders.

\section{HISPAMERICA \\ Revista de Literatura \\ Dirige Saül Sosnowski}

Año II

Nos 4-5

Diciembre 1973

SUMARIO: ENSAYOS: Walter Mignolo, "La escena y la escritura (Una hipótesis de trabajo sobre la poética en América Latina)"; Gerardo Mario Goloboff, "Javier Heraud: La palabra en su límite"; María Luisa Bastos, " 'Contorno', 'Ciudad', 'Gaceta literaria': Tres enfoques de una realidad"; Richard Reeve, "El primer cuento de Carlos Fuentes: 'Pastel Rancio'." Serie "Los marginados", III. Caso Rubén Bareiro Saguier. Entrevista: Joseph Sommers a Juan Rulfo. Poesía: Ernesto Cardenal, "Viaje a Nueva York (pocma-reportaje)". Ficción: César Fernández Moreno, "Franz, Ella y Tomás"; Ernesto Sábato, "Abadón" (fragmento) ; Iverna Codina, "Homenaje a Rackefeller". Reseñas: Bernardo Verbitsky, Etiquetas a los hombres (Saúl Sosnowski); Eugenia Echeverría, Cambio de palabras (David Lagmanovich); Arturo Carrera, Escrito con un nictógrafo (I. Oren); Angel Rama, La generación crítica: 1939-1969. I: Panoramas (Jaime Rest) : Rudolfo Anaya, Bless me, Ultima (Teresinha Alves Pereira).

Suscripciones: Estudiantes: US $\$ 5$ por año, no-estudiantes: US $\$ 10$. Suscripciones, colaboraciones, libros a Saúl Sosnowski, Hispamérica. 4330 Hartwick Road, Apt. 608. College Park, Md. 20740 U.S.A. 


\section{JOURNAL OF SPANISH STUDIES}

\section{TWENTIETH CENTURY}

Editors: Vicente Cabrera and Luis González-del-Valle

Book Review Editor: Evelio Echevarría

Editorial Advisory Council: Jaime Alazraki, Fernando Alegría, Robert L. Bancroft, Pedro Barreda Tomás, Mary Ann Beck, Harold L. Boudreau, Calvin Cannon, Rodolfo Cardona, Homero Castillo, Robert L. Coon, Ernerto Guerra Da Cal, Andrew P. Debicki, Kathleen M. Glenn, Antolín Gon. zález-del-Valle, Sumner Greenfield, Paul Ilie, Leon Livingstone, Juan Loveluck, Barnett A. McClendon, George McMurray, José Otero, Anthony M. Pasquariello, Hugo Rodríguez-Alcalá, Ivan A. Schulman, and Mario J. Valdés.

Some Pullished and Forthcoming Articles: On Arrabal, Azorín, Borges, Cortázar, Donoso, Fuentes, García Pavón, Goytisolo, Gutiérrez Nájera, Hernández, Larreta, Lugones, A. Machado, Martín Santos, Rulfo, Salazar Bondy, Urbina, and Valle-Inclán.

Book Reviews: The Journal is publishing reviews of works within the fields of Spanish and Spanish American literatures in this century. The book reviews normally should be between 2 and 4 typewritten pages and may be written in. English or Spanish. The original -with one copy- and return postage are requested. Book reviews, works to be reviewed and readers are welcome.

General Information: The Joumal publishes scholarly articles dealing with the literatures of Spain and Spanish America in this century. The periodical appears three times annually (Spring, Fall, and Winter). Manuscripts are welcome. They should be between 12 and 25 typewritten pages and prepared in accordance with the MLA Style Sheet, all notes at the end. The original, an abstract, and two additional copies of both must be accompanied by return envelope and postage. Manuscripts may be written in English or Spanish.

Address: Direct all manuscripts —studies and book reviews - to: Editors, Journal of Spanish Studies: Twentieth Century, Department of Foreign Languages, Colorado State University, Fort Collins, Colorado 80521 U.S.A. Correspondence on subscriptions, advertisements and exchanges should be sent to: Editors Journal of Spanish Studies: Twentieth Century, Department of Modern Languages, Kansas State University, Eisenhower Hall, Manhattan, Kansas 66506 U.S.A. 


\section{p I u r a I}

CRITICA Y IITERATURA

Revista mensual de Excélsior, Cía. Editorial, S. C. L. Director: Octavio Paz

Jefe de Redacción: Kazuya Sakai

Oficina: Reforma 12-505, México 1, D. F. México

Subscripciones para Estados Unidos y Canadá:

Un año (doce números) correo ordinario: US dlls. 7.50

Un año (doce números) correo aéreo: US dlls. 12.00

Subscripciones: Reforma 18, México 1, D. F. México. 


\section{INDICES DE CUADERNOS AMERICANOS}

\section{POR MATERIA Y AUTORES}

(1942-1971)

Contendrá más de 500 páginas encuadernación de lujo

Edición limitada

Se reciben pedidos previo pago

\section{PRECIOS:}

México

América y España

Europa y otros Continentes ...
$\$ 150.00$ M.N.

$13.50 \mathrm{Dls}$.

$15.00 \mathrm{Dls}$.

(EN PRENSA)

\section{CBA EDITORES}

\section{REVISTERO}

Todas las publicaciones periódicas de AMERICA LATINA fichadas, comentadas, con tarifas de suscripción, contenido y colaboradores.

Edición 1973: US $\$ 15.00$

\section{GUIA CBA 1973-74 DE LATINOAMERICA}

TODOS LOS DATOS DE REFERENGIA sobre todas las academias, asociaciones, colegios, archivos, bibliotecas, universidades, institutos, deptos. experimentales, conservatorios, museos editores diarios, radios, televisiones, servicios universitarios, sociedades de estudio e investigación, etc. de TODA LATINOAMERICA.

$350 \mathrm{pp} . \quad$ US $\$ 20.00$

PEDIDOS A: EDUARDO DARINO, EDITOR

GASILLA CORREO 1677. MONTEVIDEO URUGUAY.

NO ENVIE NUNGA CHEQUES A ESA DIREGGION, SALVO QUE SEA POR GORREO AEREO CERTIFICADO. 


\section{BULLETIN HISPANIQUE}

Revue trimestrielle, organe des hispanistes français ouvert à la collaboration étrangère

Comité Directeur:

Président: M. Bataillon, membre de l'Institut (Collège de France) Secrétaire-Gérant: N. Salomon (Faculté des Lettres de Bordeaux) Secrétaire-adjoint: M. Chevalier (Faculté des Lettres de Bordeaux)

Membres: Ch. V. Aubrum (Sorbonne)

P. Mérimée (Faculté des Letres de Toulouse)

R. Ricard (Sorbonne)

A. Rumeau (Sorbonne)

et Le Directeur des Annales, Doyen de la Faculté des Lettres et Sciences Humaines de Bordeaux

$$
\text { Abonnements: France } \ldots \ldots \ldots \ldots 66060 \mathrm{~F} \text {. }
$$

Le montant des abonnements, des demandes de numerós ou de tirages à part, les réclamations pour manques doivent être adressés à: Éditions BIERE, 18 à 22, rue du Peugue, 33000 Bordeaux (C. C. P. Bordeaux 4388.38 S.)

SIN NOMBRE

REVISTA TRIMESTRAL LITERARIA

Publicada por Editorial Sin Nombre, Inc.

Apartado 4391

San Juan, Puerto Rico 00905

Directora: Nilita Vientós Gastón

Vol. II, No. 4,

(Homenaje a Baroja)

Vol. III, No. 1

(Homenaje a Neruda)

Suscripción anual $\ldots \ldots \ldots \ldots \ldots . \$ 10.00$

Estudiantes Puerto Rico ....... $\quad 5.00$

Ejemplar suelto ........... 2.75 


\section{The Official Quarterly of the \\ American Association of Teachers \\ of Spanish and Portuguese \\ HISPANIA}

Published in March, May, September and December. Subscription, $\$ 8.00$ a year. For sample copies and information about subscription and membership in the Association, write to the Secretary-Treasurer, Eugene Savaiano, Wichita State University, Wichita, Kansas 67208. Articles and news: Editor, Donald W. Bleznick, Romance Language

Dept., University of Cincinnati, Cincinnati, Ohio 45221.

Books for review: Myron I. Lichtblau, Romance Language

Dept., Syracuse Univ., Syracuse, N. Y. 13210.

Advertising rates: Albert Turner, Glenbrook South High School, 4000 West Lake Avenue/Glenview, Illinois 60025.

\section{$\mathrm{N} O \mathrm{R}$ T E}

Revista Hispánica de Amsterdam

No. 1-2 1974: número monográfico sobre AUGUSTO ROA BASTOS

\section{Colaboraciones de:}

Augusto Roa Bastos, Jan Lechner, Jean Andreau, Enric Miret, Gerald Martin, Rubén Bareiro Saguir, Wolfgang A. Luchting.

64 págs.; precio $f$ 15. - (Holanda: $f$ 10, Suscripción anual $f 25$. - ( , $f 20$,

Pedidos: Redacción "NORTE", Postbus 297, Amsterdam, Holanda. 


\section{BOOKS ABROAD}

\section{AN INTERNATIONAL LITERARY QUARTERLY}

Founded in 1927

A QUARTERLY REVIEW AND CRITICAL ANALYSIS in English of contemporary world literature $B O O K S A B B O A D$ offers in each issue a review section that examines and evaluates the latest in poetry, drama, the novel, criticism and scholarship from as many as sixty languages, ranging from French, Spanish, Italian, German, Russian and Japanese to Romansh Catalan, Occitan, Albanian, Gaelic, Welsh, Macedonian and Frisian.

THE RESOURCES OF 600 REVIEWERS, among them the leading critics and scholars of their field, insure BOOKS ABROAD readers a professional and discriminating view of contemporary literature.

A SECTION OF ARTICLES AND COMMENTARIES balance the breadth and diversity of the review section with in-depth analyses and evaluations of new and important writers, works, literary movements and critical trends.

Individual subscription rates: $\$ 8.00$ a year; $\$ 14.00$ two years; single copies $\$ 2.50$. Address the Circulation Manager, BOOKS $A B R O A D$.

UNIVERSITY OF OKLAHOMA PRESS

Norman, Oklahoma 73069

\section{RAZON Y FABULA \\ REVISTA DE LA UNIVERSIDAD DE LOS ANDES}

Bogotá, Colombia, S. A.

No. 35, Enero-Marzo 1974

\section{S U M A R I O}

César Vallejo y España, Juan J. Gilabert.

La nueva novela hispanoamericana y la función del escritor, Ignacio Iglesias.

Todo lo que está pasando, Gustavo Mejía.

Parentesco ritual entre los indios guambianos, Oscar Osorio Gómez.

La politica internacional y la piratería en el siglo XVII, John M. Parry.

El palamedes de Eurípides y los errores judiciales en Atenas, Jouzas Zaranka.

Dedicaciones, Javier Hernández.

Crónica universitaria.

Notas bibliográficas.

Suscripción anual en Colombia $\$ 50.00$.

En el exterior US 10.00 .

Universidad de los Andes - Apdo. Aéreo 4976

Bogotá, Colombia, S. A. 


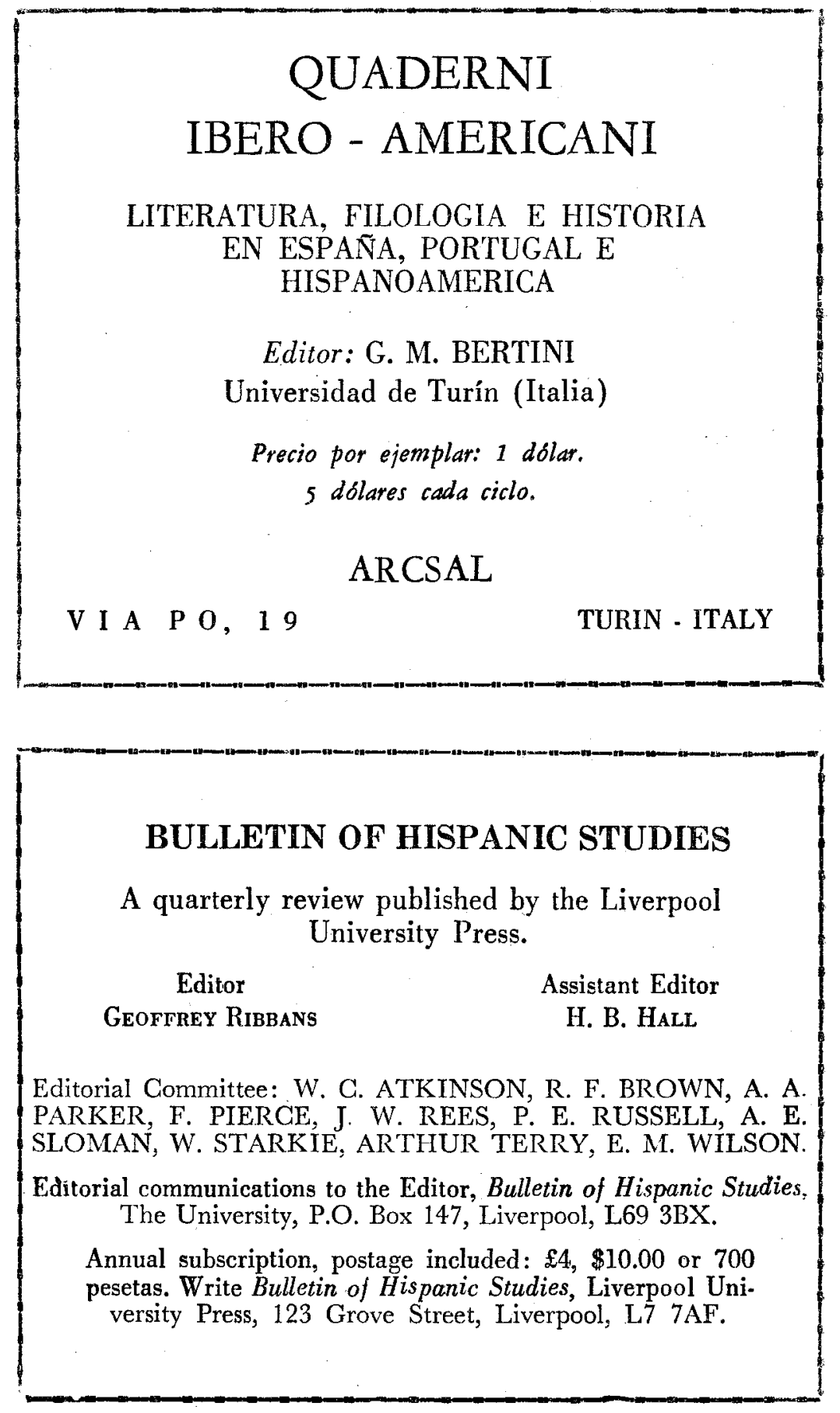




\section{TRAVAUX DE L'INSTITUT D'ETUDES LATINO-AMERICAINES DE L'UNIVERSITE DE STRASBOURG (TILAS, $\mathrm{X}, 1970$ )}

Table des matières

Activités de l'Institut.

Anniversaire par Georges LIVET

\section{LITTERATURE}

DEVOTO, Daniel: Seis poemas de Eguren.

LABERTİT, André: Exercices de style et lecture de Gongora au Pérou vers 1660

MARTINENGO, Alessandro: Curiosidad e interés americanista en Giacomo Leopardi.

SARAIVA, A. J.: Le prédicateur, Dieu et son peuple à Bahia en 1640: étude d'un sermon du Père Vieira.

\section{LINGUISTIQUE}

NANDRIS, Octavian: Ibéro-roman et ibéro-américain: problèmes de croisement linguistique et de "fonds générateur".

POTTIER, Bernard: Publications récentes sur l'espagnol et le portugais en Amérique (1965-1969).

III. HISTOIRE

AYMES, Jean-René: La connaissance du Mexique en France pendant le Consulat et l'Empire.

IV. GEOGRAPHIE

TRICART, Jean: Etude du milieu physique: quelques données méthodologiques à partir de l'exemple du Bassin du Rio Combeima (Colom. bie).

V. ETHNOGRAPHIE

PIQUERO-MOREAU, Dorita: Mythologie et symboles dans "Juan Girador" de Miguel Angel Asturias.

VI. DOCUMENTS

DUVIOLS, J. P.: "Relation des voyages du Sr. d'Accarette dans la rivière de la Platte et de là par terre au Pérou et des observations qu'il y a faites", suivies de deux mémoires inédits du même auteur pour la "Conqueste de Bonnes-Ayres dans la Rivière de la Platte en l'Amérique Méridionalle" (Edition).

\section{PUBLICATIONS}

Fonds américaniste ancien de la Bibliothèque Nationale et Universitaire (B.N.U.) de Strasbourg, 220 p. $24 \times 27 \mathrm{~cm}$. Strasbourg, 1968.

Hommage de l'Université de Strasbourg à Miguel Angel Asturias. Travaux de l'Institut d'Etudes Latino-Américaines: TILAS VIII (Supplément), 1968.

Adresse: Faculté des Lettres et Sciences Humaines de Strasbourg 25, rue du Soleil 67-Strasbourg. FRANCE. 


\title{
REVISTA HISPÁNICA MODERNA
}

\author{
Fundador: Federico de Onís
}

Se publica trimestralmente. Dedica atención preferente a las literaturas española e hispanoamericara de los últimos cien años. Contiene artículos, reseñas de libros, textos y documentos para la historia literaria moderna y una bibliografía hispánica clasificada. Publica periódicamente monografías sobre autores importantes con estudios sobre la vida y la obra, una bibliografía, por lo general completa, y unas páginas antológicas.

\section{Editor}

KARL-LUDWIG SELIG

Precio de subscripción y venta:

8 dólares norteamericanos al áño. Número sencillo 2.00 dólares; número doble: 4.00 dỏlares.

Hispanic Institute in the United States

Columbia University

612 West 116 Street

New York, 10027, N. Y.

\section{LATIN AMERICAN LITERARY REVIEW}

Revista dedicada a la literatura y el arte de América Latina y de los latinoamericanos en los Estados Unidos. Contiene monografías, reseñas de obras literarias recientes, ensayos sobre arte y traducciones de literatura creativa (poesía, cuento, drama). Publicada en inglés, dos veces al año. Subscripción \$7.00. Editores: Yvette Miller, Edward Dudley, Carlos Navarro. Dirección: Depart ment of Modern Languages, Baker Hall, Carnegie-Mellon University, Pittsburgh, Pennsylvania 15213, U.S.A. 


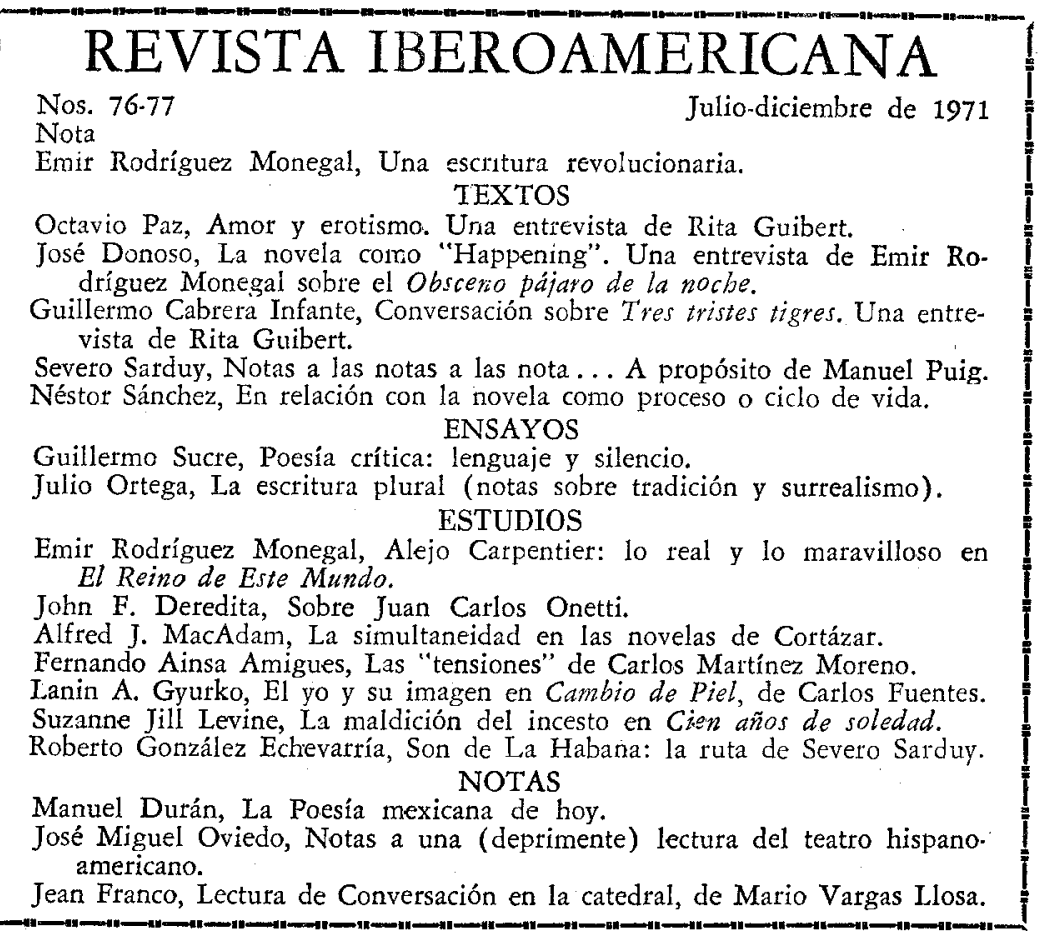

\section{HISPANIC REVIEW}

A Quarterly Journal Devoted to Rescarch in the Hispanic Languages and Literatures

Published by the Department of Romance Languages University of Pennsylvania

General Editor: Russell P. Sebold.

Managing Editor: José M. Regueiro.

Editors: S. G. Armistead, P. G. Earle, P. M. Lloyd, G. Sobejano. Associate Editors: D. L. Catron, A. E. Foley, G. Gullón, E. Seiver. Subscription: $\$ 12.00$ per year

Williams Hall, University of Pennsylvania Philadelphia, Pa. 19174 


\section{THE BILINGUAL REVIEW / LA REVISTA BILINGÜE}

A journal dedicated to the study of the linguistics and literature of English-Spanish bilingualism in the United States.

The Bilingual Review/La Revista Bilingüe functions as:

- A research and scholarly journal.

- A bibliographic resource.

- A literary magazine.

- An information outlet for job and training opportunities.

Manuscripts are encouraged. Also all information regarding job opportunities and training programs in the field of bilingualism will be published by the journal gratis. Send information and research or literary manuscripts to:

Prof. Gary Keller, editor in chief

The Bilingual Review/La Revista Bilingue.

Department of Romance Languages

City College of New York

New York, New York 10031

Volume i

May-August 1974

Number 2

\section{RESEARCH AND CRITICISM / INVESTIGACIONES Y CRITICA}

ANTHONY GIRARD LOZANO, Grammatical Notes on Chicano Spanish; NILA.MARRONE, Investigación sobre variaciones léxicas en el mundo hispano; J. S. BERNSTEIN, The Semantics of Emphasis: 'Act' and 'Scene' in English and Spanish; RANDOLPH D. POPE. Dos novelas álbum: Libro de Manuel de Cortázar y Figuraciones en el mes de marzo de Díaz Valcárcel; JUDITH GINSBERG; Los juicios de José Martí acerca de la inmigración a los Estados Unidos.

\section{LITERATURE/LITERATURA}

El Huillacoche, The Man Who Invented the Automatic Jumping Bean.

\section{REVIEW ARTICLES / RESENAS}

EUGENIO CHANG-RODRIGUEZ, Lengua y cultura en Puerto Rico [Transculturación e interferencia lin qǘsistica en el Puerto Rico contemporáneo 1898/1968, Germán de Granda]; GABRIELLA DE BEER, Language Policy in Mexico: De Jure and De Facto [Telling Tongues: Language Policy in Mexico, Shirley B. Heath]; MARIA INES LAGOS DE POPE, Una alegoria del neocolonialismo [Temporada de duendes, Pedro Juan Soto].

The journal will publish articles or fiction in any major language. It is published in October, February and May. Suscription rates: one year $\$ 6.00$; two years, $\$ 11.00$; three years, $\$ 15.00$. 


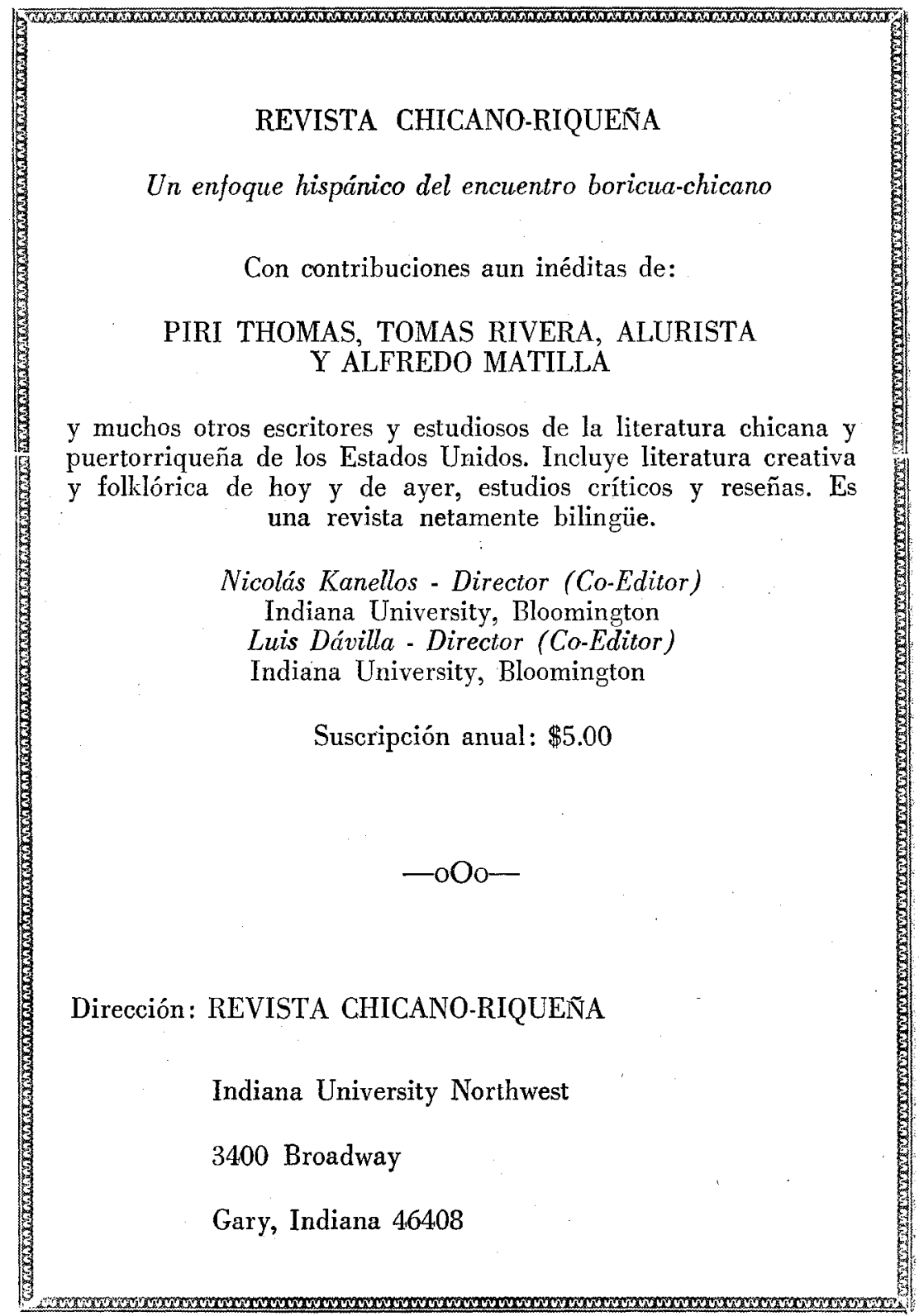




\section{ESTUDIOS CUBANOS/CUBAN STUDIES}

Estudios Cubanos/Cuban Studies (anteriormente Boletín de Estudios sobre Cuba/Cuban Studies Newsletter) es una revista académica multidisciplinaria dedicada totalmente a Cuba. Se publican dos números al año, en enero y julio, que regularmente incluyen:

- artículos sobre temas contemporáneos

- bibliografía clasificada de publicaciones recientes

- anotaciones de libros importantes

* inventario de investigación en curso

Algunos de los artículos que aparecerán en próximos números son

- Desarrollo y aplicación de las ciencias de computación e informática

- El papel cambiante de los militares

- La asimilación de cubanos exilados en los EE. UU.

Números anteriores han incluido ensayos bibliográficos sobre la obra de Fidel Castro, la mujer cubana en el siglo $\mathrm{xx}$, literatura creativa en la isla y el exilio, lista de revistas y periódicos, y de tesis doctorales escritas en EE.UU. sobre Cuba.

Estudios Cubanos/Cuban Studies se puplica por el Centro de Estudios Latinoamericanos, Centro Universitario de Estudios Internacionales, Universidad de Pittsburgh. La subscripción anual es de $\$ 4.00$ (dólares U.S.A.) si individual, y $\$ 10.00$ si por instituciones (vr. gr., bibliotecas). Los números atrasados cuestan $\$ 2.00$ a individuos y $\$ 3.00$ a instituciones.

Enviar la correspondencia a: Center for Latin American Studies, University of Pittsburgh, 216 Mervis Hall, Pittshurgh, PA 15260, EE.UU. 
